\title{
Cultures visuelles et révolutions : enjeux et nouvelles problématiques
}

Guillaume Mazeau, Pascal Dupuy, Charlotte Guichard, Richard Taws et Pascal Griener

\section{Q OpenEdition \\ 12 Journals}

\section{Édition électronique}

URL : https://journals.openedition.org/ahrf/12794

DOI : $10.4000 /$ ahrf.12794

ISSN : 1952-403X

Éditeur :

Armand Colin, Société des études robespierristes

\section{Édition imprimée}

Date de publication : 1 juin 2013

Pagination : 143-160

ISBN : 978-2-9083-2789-2

ISSN : 0003-4436

Référence électronique

Guillaume Mazeau, Pascal Dupuy, Charlotte Guichard, Richard Taws et Pascal Griener, « Cultures visuelles et révolutions : enjeux et nouvelles problématiques », Annales historiques de la Révolution française [En ligne], 372 I avril-juin 2013, mis en ligne le 01 juin 2016, consulté le 01 juillet 2021. URL : http://journals.openedition.org/ahrf/12794 ; DOI : https://doi.org/10.4000/ahrf.12794 
REGARDS CROISÉS

\title{
CULTURES VISUELLES ET RÉVOLUTIONS : ENJEUX ET NOUVELLES PROBLÉMATIQUES
}

\author{
Guillaume MAZEAU \\ Pascal DUPUY \\ Charlotte GUICHARD \\ Richard TAWS \\ Pascal GRIENER
}

Parce que la question des liens entre les cultures visuelles et les révolutions est aujourd'hui en profond renouvellement, nous avons souhaité présenter quelques-uns des débats qui traversent un champ en pleine expansion mais qui reste pourtant encore très méconnu ou sujet à de nombreux malentendus, notamment en France.

Méconnues des historiens, les images de «la » Révolution ? Cette affirmation peut surprendre. Depuis les années 1980, de nombreux travaux ont en effet totalement transformé la place des images dans l'histoire des révolutions. Si elles sont encore loin de s'être banalisées, si elles sont encore trop souvent traitées comme de simples illustrations ou comme des sources de second ordre, si l'analyse iconographique reste encore peu enseignée et pas assez pratiquée, les images sont néanmoins entrées de plein droit dans les corpus utilisés par les historiens.

Entrepris dans un contexte d'essor de l'histoire des représentations et de la fondation du Musée d'histoire de la Révolution française de Vizille en 1983, les travaux menés avant et après le Bicentenaire par Michel Vovelle, Antoine de Baecque, Claude Langlois, Annie Duprat, Philippe Bordes, Ronald Paulson, Philippe de Carbonnières au musée Carnavalet, Rolf Reichardt, James A. Leith, Ian Germani, Annie Jourdan, Claudette Hould ou Pascal Dupuy témoignent de la vitalité de cette histoire politique des images des périodes révolutionnaire et napoléonienne, notamment centrée sur les tableaux, les allégories, les gravures et les caricatures.

Dans le contexte de développement des Cultural Studies ou de la New Cultural History, posant les questions de la violence, du corps et 
du genre, d'autres historiens et historiennes s'employèrent quant à eux à intégrer les images en pratiquant l'interdisciplinarité et en s'ouvrant ainsi aux vastes champs de l'histoire anthropologique, politique et culturelle. Les travaux de Lynn Hunt, concrétisés par le projet digital « Imaging the French Revolution » (avec Jack Censer), les études de Ludmilla Jordanova, Joan B. Landes et Leora Auslander mais aussi, en France, d'Anne Lafont ont ouvert de nouvelles perspectives permettant de repenser non seulement le rôle des images, mais plus largement de la culture visuelle dans les changements politiques et sociaux de la seconde moitié du XVIIIe siècle. Contemporains des nouvelles réflexions de l'histoire de l'art portant sur les milieux et pratiques artistiques (Thomas Crow), le marché de l'art et le jugement de goût, en relation avec les développements des sciences cognitives (Barbara Maria Stafford), ces travaux, souvent associés au courant des Visual Studies ${ }^{1}$, interrogent les diverses manières dont les individus voient le monde (le «régime scopique ») et/ou sont vus en fonction de leur contexte politique, social, culturel et de leur propre historicité. Situés au croisement de plusieurs disciplines, ces nouveaux chantiers analysent les pratiques et cultures visuelles à travers les estampes, mais aussi les textes, les illustrations, le mobilier, les vêtements ou les instruments d'optique, renouvelant considérablement la compréhension du rôle des signes quotidiens dans l'histoire des révolutions.

Afin de discuter de l'intérêt et, peut-être, des limites de ces thématiques, pour étudier les révolutions de la fin du XVIII ${ }^{\mathrm{e}}$ siècle, nous avons demandé à trois spécialistes de répondre à nos questions. Professeur à l'Université de Neuchâtel, Pascal Griener est notamment l'auteur du livre La République de l'œil. L'expérience de l'art au siècle des Lumières (Paris, O. Jacob, 2010), dans lequel il étudie les transformations du regard sur l'art au Siècle des Lumières. Chargée de recherches au CNRS (UMR 8529, CNRS/Université Lille-3), Charlotte Guichard a étudié le rôle de la figure de l'amateur d'art dans son livre Les amateurs d'art à Paris au XVIII ${ }^{e}$ siècle (Champ Vallon, 2008). Enfin, Richard Taws, Lecturer [Maître de conférences] en Histoire (University College, Londres), s'est intéressé à la manière dont la culture visuelle éphémère a participé à la création d'une nouvelle culture politique dans The Politics of the Provisional. Art and Ephemera in Revolutionary France (Pennsylvania State University Press, 2013).

(1) Pour cerner les enjeux et les méthodes de ce courant, on peut se reporter à « Visual culture questionnaire », October, n 102 , été 1996, p. 25-70. 
Guillaume Mazeau (Université de Paris I-Panthéon-Sorbonne/IHRF) et Pascal Dupuy (Université de Rouen/GRHIS)

Dans quelle mesure peut-on dire que la manière de voir le monde change-t-elle dans la seconde partie du XVIII siècle?

\section{Pascal Griener}

La question ne m'est pas totalement claire, mais elle postule implicitement la corrélation directe entre révolution scientifique régionale (au sens de région du savoir) et révolution politique. Or cette corrélation n'est pas avérée. La révolution du regard que je décris dans mon livre (La République de l'œil. L'expérience de l'art au siècle des Lumières, Paris, O. Jacob, 2010) porte spécifiquement sur le regard sur l'art, qui se transforme profondément au siècle des Lumières ; cependant, il ne s'agit pas d'un développement organique, harmonieux, mais d'une évolution irrégulière, saccadée et parcourue de tensions graves. Personnellement, je me méfierais de la notion de vision du monde (Weltanschauung), qui a été justement remise en cause il y a de nombreuses décennies, parce qu'elle attribue à une entité collective une « personnalité » comme une " pensée » unique. D'autre part, l'émergence de la philosophie critique, autrefois considérée comme un phénomène caractéristique du XVIII ${ }^{\mathrm{e}}$ siècle (Reinhart Koselleck ${ }^{2}$ ), est aujourd'hui saisie dans la plus longue durée (Jonathan Israel ${ }^{3}$ ). Ce qu'on appelle les Lumières radicales se construit dès le XVII ${ }^{\mathrm{e}}$ siècle avec Spinoza, au cœur d'une Europe où domine la monarchie absolue. La culture expérimentale se développe durant la même période. Le champ de l'art, qui me concerne plus particulièrement, n'a bénéficié de ces apports qu'avec quelque retard.

\section{Richard Taws}

Il s'agit d'une question à laquelle il est difficile de répondre sans paraitre désinvolte. Je dois avouer que de manière générale, je me méfie des tentatives d'universalisation visant à caractériser des transformations considérables dans la manière de voir les choses à travers une période historique donnée. Ce type d'approche risque d'aplanir les complexités de l'expérience sensorielle individuelle, tout en simplifiant la diversité de la pratique visuelle et les récits de la période historique qu'elle prétend éclairer. À n'en pas douter, pour éviter de faire abstraction à tort des particularités

(2) Reinhart KosellecK, Kritik und Krise. Eine Studie zur Pathogenese der bürgerlichen Welt, Freiburg, Karl Alber Verlag, 1959.

(3) Jonathan ISRAEL, Radical Enlightenment: Philosophy and the Making of Modernity (1650-1750), Oxford, Oxford University Press, 2001. 
liées aux considérations de classe, de genre ou de race, ou d'omettre des déterminants plus précis, il convient de se demander en premier lieu «qui sont les observateurs ? » et « que ou qui regardent-ils ? » Ceci dit, il me semble qu'il est possible de discerner certains changements dans la manière dont le monde a été abordé sur le plan visuel dans la seconde partie du dix-huitième siècle, et que ces changements pourraient être liés, de manière complexe et sans rapport nécessaire de cause à effet, aux transformations de la vie sociale et politique qui ont été observées au cours de cette période.

Les chercheurs qui travaillent dans le domaine des « études visuelles » [en anglais visual studies] ou s'en approchent, soulignent depuis un certain temps l'importance d'envisager la perception visuelle comme un phénomène spécifique sur le plan historique. Ils soulignent combien la «culture visuelle » ne consiste pas simplement à ajouter une panoplie apparemment sans fin d'images et d'objets, souvent issus de la culture populaire, à un canon existant de la peinture, de la sculpture ou de l'architecture. Certains avancent plutôt qu'une approche sérieuse des études visuelles requiert de porter son attention sur les nouvelles manières dont la population voit le monde et sur le croisement des images, de l'optique, des institutions et des textes qui ont tous été historiquement déterminés. Cet argument a été défendu avec conviction à propos de la culture spectaculaire du début du dix-neuvième siècle, avec ses passages, ses panoramas et l'efflorescence de nouveaux produits artistiques revendiquant leur propre histoire. Cette caractéristique s'applique également à la fin du dix-huitième siècle, lorsque la connaissance a été réorganisée en termes visuels et lorsque la politique et l'art ont commencé à s'entremêler de plus en plus. Ce phénomène n'est bien entendu pas propre à la France. L'évolution des sciences, ou l'émergence de l'esthétique en tant que discours indépendant au sein de la philosophie en Allemagne et en Grande-Bretagne, par exemple, témoignent également d'une refonte significative des possibilités présentées par la perception visuelle lors du Siècle des Lumières en Europe. Toutefois, les changements d'ordre social et politique en France ont joué un rôle crucial dans ces discours et la question demeure : dans quelle mesure la Révolution française peut-elle être considérée comme une phase de transition importante dans l'histoire de la perception visuelle ? Malgré une angoisse rousseauiste relative au statut des images qui ont façonné des approches révolutionnaires de la perception visuelle, les images ont joué un rôle significatif dans la Révolution, non seulement en matière de documentation des événements que nous pouvons estimer diversement proches ou distants de la manière dont nous comprenons leur déroulement, 
mais également comme élément crucial du processus historique en luimême.

Je dirais en effet que les images datant de cette période peuvent constituer la meilleure manière de formuler des suppositions de large portée relatives aux transformations du regard. Les images portent souvent les traces de leur utilisation et de leurs abus, et elles offrent la possibilité de combler le fossé entre une culture « visuelle » et «matérielle». Il est crucial de prendre en compte non seulement ce que la culture visuelle de la Révolution française représentait, mais également comment elle le représentait et ce que cette représentation a rendu possible (ou impossible). Ce type d'approche implique nécessairement un élargissement de la portée de la perception visuelle en prenant en compte un éventail de matériaux qui ne sont pas généralement pris en considération dans le cadre de l'art. Cet élément revêt une importance particulière dans le contexte de la fin du dix-huitième siècle, alors que les hiérarchies caractérisant les « beaux-arts » et les « arts populaires » étaient de plus en plus remises en question.

\section{Charlotte Guichard}

Il est difficile de dater précisément un changement global dans la manière de voir le monde car les temporalités de la culture visuelle sont multiples. Un certain nombre de déplacements sont perceptibles dès la fin du XVII ${ }^{\mathrm{e}}$ siècle, au point que certains historiens, comme Peter Burke, ont parlé à ce propos de «tournant visuel ». Dans les sciences de l'observation et de la description, dont relèvent aussi bien l'histoire de l'art que l'histoire naturelle, l'image n'a plus un statut seulement illustratif ou ornemental, elle fait preuve dans l'argumentation scientifique et revêt une dimension cognitive. La formule choisie par Burke s'applique à l'origine aux cultures antiquaires, qui se détournent de l'approche philologique, fondée sur l'étude des textes, pour s'appuyer désormais sur les «monuments ». Dès lors, c'est l'image qui va concrètement ordonner la démonstration et le récit : l'antiquaire Bernard de Montfaucon formalise ce nouveau statut de l'image dans les savoirs dans sa préface à L'Antiquité expliquée et représentée en figures (1719-1724) « Je réduis dans un corps d'ouvrage toute l'antiquité ; par l'antiquité, j'entends seulement ce qui peut tomber sous les yeux \& ce qui se peut représenter dans des images $»$. Ce tournant visuel a un impact décisif sur la manière de faire l'histoire des sociétés européennes et joue un rôle aussi dans les réflexions sur les archéologies urbaines ${ }^{4}$. Dans le

(4) Peter BURKE, «Images as Evidence in Seventeenth-Century Europe », Journal of the History of Ideas, 62-2, 2003, p. 272-296; Alain SCHNAPP, La conquête du passé. Aux origines 
domaine artistique, les œuvres peintes et sculptées, conservées dans des collections privées, sont reproduites par la gravure dans des recueils et des «musées de papier», ainsi promises à une divulgation plus grande. Une culture du débat s'ouvre grâce à la circulation européenne de ces livres d'images au sein des réseaux de savants et d'amateurs : l'imaginaire visuel des Lumières en est durablement affecté et contribue en retour à des débats essentiels autour de la définition de l'idée de civilisation (Winckelmann) ${ }^{5}$. Mais le tournant visuel s'applique également à l'histoire naturelle : la forme « atlas » émerge comme lieu privilégié de la mise en recueil des images scientifiques (anatomie, botanique, géographie). Dans un ouvrage consacré à l'histoire de l'objectivité scientifique, Lorraine Daston et Peter Galison ont montré les enjeux épistémologiques que soulève la prégnance de l'image dans le domaine des sciences de l'observation et de la description, et ce dès la fin de l'âge classique. Au XVIII siècle, l'avènement de l'image synthétique et raisonnée, dont la botanique de Carl Von Linné est exemplaire, participe au déploiement théorique de l'objectivité scientifique et à ses dispositifs pratiques. Les atlas font la science : véritables « dépositaires des images d'archives du monde empirique $»^{6}$, ils contribuent activement aux différents régimes épistémiques. Au XVIII ${ }^{\mathrm{e}}$ siècle, la description du monde devient essentiellement visuelle : il y a donc une vérité de l'image, aussi bien pour décrire les artefacts que le monde naturel. La nouvelle efficacité cognitive de l'image est alors fondée sur une étroite articulation entre un contenu théorique qui guide les codes de la représentation ( « vérité d'après nature ») et des dispositifs matériels qui sont à l'origine de la fabrication de l'image.

Quel est le rôle des nouvelles techniques graphiques et des progrès optiques dans ce changement?

\section{Charlotte Guichard}

L'importance du XIX ${ }^{\mathrm{e}}$ siècle dans l'avènement d'un nouveau « régime scopique » lié aux révolutions technologiques de l'image (Jonathan Crary), a occulté l'importance des innovations techniques et optiques du siècle des Lumières pour une histoire du regard. Celles-ci ont pourtant été

de l'archéologie, Paris, Carré, 1993 ; Stéphane Van DAMME, Métropoles de papier. Naissance de l'archéologie urbaine à Paris et à Londres (XVII $-X X^{e}$ siècle), Paris, Les Belles Lettres, 2012.

(5) Élisabeth DÉCUlTOT (dir.), Musées de papier : l'Antiquité en livres 1600-1800, Musée du Louvre, Paris, 2010.

(6) Jean-Marc BESSE, Face au monde : atlas, jardins, dioramas, Paris, Desclée de Brouwer, 2003 ; Lorraine DAston et Peter GALISON, Objectivité, Dijon, Les Presses du Réel, 2012. 
un sujet important pour les historiens de l'art et en particulier les historiens de la gravure, mais qui a peu suscité l'intérêt chez les historiens de la culture. Ces innovations technologiques sont en lien avec ce que Dror Wahrman a récemment proposé d'appeler la révolution de l'information « Print 2.0 » (en référence à la révolution d'internet Web 2.0). Ce phénomène, commencé au tournant du XVIII ${ }^{\mathrm{e}}$ siècle avec l'explosion des imprimés bon marché (journaux, pamphlets, discours, gravures d'art), est l'objet des trompe-l'œil illusionnistes que réalise le peintre hollandais Edwaert Collier et qui reproduisent minutieusement cette masse d'imprimés éphémères, en jetant parfois sur eux le soupçon de l'inauthenticité7.

Par-delà la masse nouvelle des images gravées, les techniques de reproduction sont de plus en plus fines : gravures en manière de dessin et de lavis qui suscitent l'illusion du dessin et de l'aquarelle, gravures en couleurs et lithographies à la fin du siècle. Dans le domaine des beaux-arts, l'invention du fac-similé (gravure fidèle aux dessins des maîtres anciens) facilite l'essor des pratiques d'attribution et une archéologie du faire fondés sur le dessin ${ }^{8}$. Ces inventions pourraient paraitre réservées aux spécialistes mais elles favorisent aussi une synergie entre inventeurs d'instruments et utilisateurs ou spectateurs, qui suscite des consommations culturelles nouvelles liées à l'image, comme les lanternes magiques, les panoramas ou les physionotraces qui se développent à la fin du siècle avec un marché en plein essor.

Images, savoirs et culture technique de l'instrument entrent donc dans un dialogue fécond dans la seconde moitié du XVIII ${ }^{\mathrm{e}}$ siècle au point de nourrir une nouvelle culture du diagramme («culture of diagram») qui innerverait la visualisation de la connaissance dès la publication des planches de l'Encyclopédie. L'image, structurée par des éléments graphiques comme points, lignes, symboles, vise à produire et à stocker la connaissance, dans un processus toujours ouvert. La théorie de l'observateur qui la sous-tend n'est pas celle qui prévaut dans le régime plus souvent évoqué de la représentation et du mimétisme ; c'est celle d'un homme capable de synthétiser l'information, en croisant des données, en les combinant à la manière de tables et de tableaux. C'est un observateur, qui, grâce à ces images graphiques, se situe entre la perception et les idées,

(7) Dror WAHRMAN, Mr. Collier's Leter Racks. A Tale of Art and Illusion at the Threshold of the Modern Information Age, Oxford, Oxford University Press, 2012.

(8) Sophie RAUX (dir.), Quand la gravure fait illusion : autour de Watteau et Boucher, le dessin gravé au XVIII siècle, Musée des Beaux-arts de Valenciennes, 2006 ; Pascal GRIENER, La République de l'œil. L'expérience de l'art au siècle des Lumières, Paris, O. Jacob, 2010. 
entre le domaine de la sensation et de l'expérience, et celui d'une culture technique, mathématique et probabiliste ${ }^{9}$. On voit ici comment la culture technique et les nouvelles machines optiques ont permis de modifier la visualisation des données et de la connaissance

\section{Richard Taws}

Cette période a été la scène d'évolutions techniques clés qui ont influencé la manière dont les personnes recevaient les informations visuelles. De l'invention de la lithographie par Alois Senefelder en 1796 aux avancées de la stéréotypie, des machines à graver de Nicolas-Jacques Conté à la popularité croissante du physionotrace, examiné par Guillaume Mazeau comme un des « nombreux appareils visuels, qui ont, discrètement, participé à l'éclosion du premier monde médiatique, entre les années 1770 et les années 1830 », les possibilités offertes par les images reproduites mécaniquement ont particulièrement modifié la manière dont les individus percevaient leur monde ${ }^{10}$. Ces transformations n'ont toutefois pas été appliquées de manière uniforme et leurs effets n'ont pas été ressentis partout de même façon. Il est selon moi particulièrement important d'éviter un déterminisme technologique irréfléchi qui rendrait trop évident le lien entre les nouvelles techniques artistiques et l'évolution historique. Bien qu'une association directe entre les récits conventionnels des « progrès » techniques et la révolution politique ou les progrès observés dans la sphère sociale soit séduisante, elle n'en reste pas moins en grande partie artificielle.

De nombreuses techniques graphiques utilisées au cours de cette période se distinguent par leur persistance, voire par leur résurrection et leur transformation sous l'impulsion de nouveaux impératifs commerciaux et politiques. Cela complique de manière significative le modèle influent du changement technique de Walter Benjamin associé aux nouvelles modalités de la perception visuelle, car comme l'ont démontré des historiens de l'art tels que Stephen Bann, si le dix-neuvième siècle a donné lieu à des innovations techniques, comme la photographie, celles-ci n'ont pas remplacé les techniques précédentes telles que la peinture ou la gravure, mais ont coexisté, souvent de manière très productive ${ }^{11}$. Il est certain que séparer trop distinctement la production visuelle de la France des années

(9) John BENDER et Michael MARrinan, The Culture of Diagram, Stanford, Cal., Stanford University Press, 2010.

(10) Guillaume MAZEAU, « Portraits de peu. Le physionotrace au début du XIX ${ }^{\mathrm{e}}$ siècle », Revue d'histoire du XIX ${ }^{e}$ siècle, 45, 2012/2, p. 35-52.

(11) Stephen BAnN, Parallel Lines : Printmakers, Painters and Photographers in NineteenthCentury France, New Haven et Londres, Yale University Press, 2001. 
1790 en termes de supports ou de techniques est potentiellement limitatif, et nous ferions peut-être mieux de porter notre attention sur la manière dont ces supports se sont chevauchés, non pas pour aplanir leurs différences, mais bien pour mieux réaliser leur spécificité par rapport aux autres formes de représentation.

\section{Pascal Griener}

L'affinement des techniques d'observation microscopique et astronomique est patent durant cette période. Il faut souligner que l'acuité de l'observation ne suffit pas à définir une révolution scientifique. Tout son « encadrement» (Ian Hacking) prend une ampleur nouvelle : au premier rang, la capacité, chez les savants, de créer des protocoles d'observation, de schématiser la réalité par des représentations graphiques opératoires, qui viennent au secours de l'œil et permettent de construire une relation plus organique entre l'observation et le concept ${ }^{12}$. Le dispositif complexe, hétérogène mais structuré qui dépose ce savoir expérimental, je l'ai nommé l'instrumentarium. C'est aussi au siècle des Lumières que s'affirme l'idée que l'expérimentation, déposée sous une forme rationnelle dans une publication imprimée, s'adresse à un auditoire universel comme instance de jugement dotée de règles reconnues de tous. Ajoutons enfin que l'image à vocation cognitive acquiert lentement le statut d'un substrat mémoriel qui permet au savant, sous certaines conditions, de travailler presque exclusivement sur des représentations. L'idée - géniale - de Quatremère de Quincy (1796) est la suivante : appliquer la théorie d'Adam Smith sur la division du travail à l'organisation de la recherche archéologique. Dans cette représentation, deux groupes assument deux tâches distinctes mais complémentaires : les uns produisent la publication savante d'objets individuels découverts, les autres synthétisent les publications illustrées produites par les premiers. Les Lumières inventent ainsi une réflexion sur les conditions épistémologiques qui assurent la validité partielle de la représentation graphique, au bénéfice d'une instance de jugement démocratique : la République des Lettres étendue à tous les domaines du savoir.

Quel est le lien entre ces changements et les transformations politiques et sociales?

(12) Christoph HofFMANN (ed.), Daten sichern: Schreiben und Zeichnen als Verfahren der Aufzeichnun, Wissen im Entwurf, 1, Zurich, Diaphanes, 2008 ; Barbara WiTTMANN (éd). Spuren erzeugen: Zeichnen und Schreiben als Verfahren der Selbstaufzeichnung, Wissen im Entwurf, 2, Zurich, Diaphanes, 2009 ; Karin KraUTHAUSEN et Omar W. NASIM (ed.), Notieren, Skizzieren, Schreiben und Zeichnen als Verfahren des Entwurfs, Wissen im Entwurf, 3, Zurich, Diaphanes, 2010. 


\section{Pascal Griener}

Cette question me semble caractéristique de l'historiographie française, qui reste obsédée par l'idée de faire coïncider révolution scientifique et Révolution française. Le problème, vu depuis l'Angleterre, l'Italie ou les états germaniques me semble perdre sa pertinence. Le développement des sciences et de méthodes du regard, dans ces pays, n'a pas conduit à la mise en cause de la réalité politique contemporaine - surtout en Angleterre et en Italie ! Même en France, un Roger Chartier a justement souligné que c'est la Révolution qui instaure elle-même l'idée de rupture. Chartier montre le danger d'une pensée qui considère cette rupture comme une réalité, déterminant comme par miracle tous les phénomènes caractérisant la culture de cette période.

On peut même invoquer un exemple plus criant de discrépance : la Révolution, en France, n'a guère favorisé le développement d'une image politique efficace, au contraire. Durant cette période de bouleversements, l'imagerie emprunte très largement son vocabulaire à l'allégorie la plus obscure. Les classes qui mènent la Révolution - aristocrates convertis, ou éléments issus des professions libérales - demeurent persuadées que l'allégorie « rationnelle » constitue un instrument éducatif idéal au service du peuple. Or, loin de constituer un langage «naturel », l'allégorie repose sur une codification complexe et arbitraire, et donc sur une initiation intellectuelle préalable, accessible aux seules élites. Paradoxalement, il faudra toute la force de Napoléon - la force d'un pouvoir personnel - pour trancher ce nœud gordien : le Premier Consul, puis l'Empereur Napoléon rejetèrent l'allégorie comme instrument de communication aux masses.

En définitive, il est impossible de dresser un bilan synthétique, en projetant un modèle progressif global sur la période. Les cultures visuelles se développent selon des enjeux précis, selon les domaines; de l'un à l'autre, la situation change profondément. Citons un exemple : la cartographie hollandaise du XVII ${ }^{\mathrm{e}}$ siècle se caractérise par une qualité scientifique exceptionnelle : elle est portée par un peuple dont les acteurs économiques connaissent l'enjeu économique et politique de renseignements géographiques exacts, à un moment où ce pays déploie un commerce international d'immense envergure. La cartographie française des Lumières - je l'ai observé en étudiant les productions des Robert de Vaugondy - en aspirant à se plier à des impératifs mondains, voire esthétiques, de sa clientèle, en arrive à créer des cartes et des globes à la valeur scientifique... inférieure !

Nous avons également tendance à étudier les Lumières selon un point de vue moderniste, qui fausse parfois l'analyse du passé. Historien d'art moi-même, j'ai étudié, comme Charlotte Guichard, les amateurs du 
XVIII ${ }^{\mathrm{e}}$ siècle. Cependant, je me rends compte que le développement de ce groupe d'acteurs culturels reste confidentiel. Par contre, d'autres arts, comme le théâtre, ont touché une immense portion de la population, toutes classes confondues. La révolution imposée par l'acteur shakespearien David Garrick au XVIII ${ }^{\mathrm{e}}$ siècle - plonger la salle dans le noir, éclairer la scène par une rampe, exclure le public de la scène - eut un impact inouï en Europe. Plusieurs arts, comme la peinture, en furent directement affectés. Ce système induisait une participation sensible décuplée chez le spectateur. Or à l'époque où ce type de dispositif s'impose, la théorie rousseauiste du théâtre séduit graduellement les publics. La fête révolutionnaire, comme lieu de célébration rousseauiste où la distinction entre acteurs et assistance n'est plus claire, et les tableaux vivants ont exploité toutes ces innovations. C'est là toute la difficulté : reconstituer une culture ancienne, et pondérer l'impact «transmédial » d'un moyen d'expression à d'autres. Bien des participants aux fêtes révolutionnaires ne comprenaient guère les symboles représentés dans les processions, mais il leur suffisait de se sentir partie prenante d'une célébration collective.

\section{Richard Taws}

Dans un contexte révolutionnaire, il est clair que certaines techniques graphiques convenaient mieux que d'autres à la documentation d'un contexte politique en plein changement. Les gravures à l'eau-forte, par exemple, pouvaient être réalisées à bien moindre prix et plus rapidement que les peintures historiques de grandes dimensions, et elles étaient mieux adaptées pour répondre directement aux événements contemporains. Certains genres ont également prospéré : la caricature, à titre d'exemple, était une forme particulièrement puissante de critique politique, même si elle se réalisait surtout en dehors du contrôle institutionnel, tandis que son homologue honorifique dans la production de portraits représentait la majeure partie des peintures exposées dans les Salons de la Révolution. Cependant, malgré les affirmations des révolutionnaires, la plupart des images de la Révolution, en termes de format, de genre et parfois d'iconographie, existaient déjà plus tôt au dix-huitième siècle, si ce n'est avant. Toutefois, si les producteurs d'images révolutionnaires se sont inspirés de traditions visuelles de longue date pour réaliser leurs œuvres, cela ne signifie pas que ces images n'étaient que des rappels de ce qui avait été fait plus tôt : ces objets méritent d'être examinés dans le contexte historique spécifique de leur production et utilisation.

En parallèle à des images explicitement « artistiques », comme je l'ai suggéré, nous devrions également aborder les nombreuses autres manières 
dont la sphère visuelle s'est transformée au cours de la Révolution française, qu'il s'agisse de l'argent, de l'habillement, des meubles, de l'équipement scientifique, des divertissements ou des documents administratifs ${ }^{13}$. En tentant de déterminer comment les contemporains de la Révolution « voyaient les choses », nous avons souvent fait fi des objets qui leur faisaient le plus se rendre compte quotidiennement des ambiguïtés découlant du visuel et de leur propre position précaire de spectateurs. Pour aborder sérieusement la culture visuelle révolutionnaire, il convient de prendre en compte une grande variété de choses réalisées pour être vues, ne faisant pas explicitement référence à des événements et à des thèmes de la révolution localisables et identifiables (des œuvres qui «illustrent » explicitement un récit historique), mais nous devons également penser au contingent, au multiple, au marginal et au central, ainsi qu'au non figuratif, au non artistique et au non révolutionnaire.

\section{Charlotte Guichard}

Il est difficile de voir un lien direct entre cette nouvelle culture visuelle et savante, marquée par une forte technicité, et les transformations politiques du siècle. Ici, le partage entre l'histoire de l'art qui s'intéresse de plus en plus aux cultures visuelles savantes dans le cadre d'un retour réflexif sur l'histoire de la discipline, et les historiens qui s'intéressent plutôt aux images vernaculaires, reste encore très net. L'étude des gravures d'actualité, bien connues en France et en Angleterre, et en particulier des caricatures, joua un rôle important dans le dialogue entre les disciplines. Depuis les travaux de Michel Vovelle, Annie Duprat et Antoine de Baecque, on mesure l'importance des caricatures révolutionnaires dans la fabrique de l'opinion publique et dans la destruction de l'Ancien Régime.

Actuellement, un nouveau point de dialogue fécond concerne les mutations du regard en lien avec l'administration impériale. Le regard porté sur le monde change car le monde a changé. Les cultures visuelles impériales sont actuellement un domaine en plein essor, notamment chez les historiens de l'art anglo-américains affectés par le tournant global de l'historiographie. Par-delà la grande peinture d'histoire, le développement des grandes explorations scientifiques et le début des empires dans la seconde moitié du XVIII ${ }^{\mathrm{e}}$ siècle suscitent la fabrication de nouvelles images, celles des Autres, que l'on trouve dans la gravure ou sur des objets décoratifs ou d'intérieur. Ces images contribuent aux débats, marqués à la

(13) Richard TAws, The Politics of the Provisional: Art and Ephemera in Revolutionary France, University Park, Pennsylvania State University Press, 2013. 
fois par l'anthropologie scientifique, les théories esthétiques et les politiques impériales, qui vont avoir d'importants impacts sur l'administration et l'exploitation économique des colonies, sur les débats autour de l'esclavage, voire aussi sur la place des Autres dans la chaîne des êtres vivants. Comme le montrent les travaux de Darcy Grimaldo Grigsby et d'Anne Lafont, la visualité est ici primordiale si l'on songe par exemple que la question de la couleur dans la représentation est éminemment politique et ne comporte pas seulement des enjeux esthétiques ${ }^{14}$.

Ces images des Autres témoignent d'une mutation du regard savant et activent différents dispositifs de représentations esthétiques, naturalistes ou anthropologiques qui vont jouer un rôle important dans l'administration des empires. Ainsi, les représentations visuelles pacifiées et séduisantes des Caraïbes anglaises à partir des années 1760 contribuent à la légitimation de l'esclavage, défendent l'exploitation économique et, même après l'abolition de l'esclavage en Jamaïque en 1830, contribuent encore à la théorie de l'inégalité des races ${ }^{15}$.

Ce nouveau regard change-t-il la place de l'individu dans la société ?

\section{Charlotte Guichard}

Dans mon travail sur l'amateur, j'ai essayé de montrer combien l'intérêt nouveau porté aux images d'art ou de sciences, aux objets naturels et aux artefacts avait modifié la manière dont la société se représente, se pense et se recompose en France au siècle des Lumières ${ }^{16}$. L' " ' amateur » est cette catégorie sociale nouvelle (le terme apparaît en 1694 dans le Dictionnaire de l'Académie française) à travers laquelle certains individus, passionnés de culture, se définissent et se représentent : c'est ainsi qu'une identité sociale se construit à partir de certaines consommations culturelles (dans lesquelles l'image a une place importante mais pas exclusive) à distance des cadres organicistes de l'Ancien Régime. Au XVIII siècle la catégorie de l'amateur est donc fortement liée à une façon nouvelle de

(14) Darcy GRIMALDO GRIGSBy, Extremities : Painting Empire in post-revolutionary France, New Haven, Yale University Press, 2002 ; Anne LAFONT, « Histoire de l'art et représentation des Noirs : la double occurrence », Lumières : L'invention et la représentation des races au XVIII ${ }^{e}$ siècle, 2010-14, p. 115-132.

(15) Kay Dian KRIZ, Slavery, Sugar, and the Culture of Refinement : Picturing the British West Indies, 1700-1840, New Haven, Yale University Press, 2008 ; Daniela BlEICHMAR, Visible Empire Botanical Expeditions and Visual Culture in the Hispanic Enlightenment, University of Chicago Press, Chicago, 2012.

(16) Charlotte GuICHARD, Les amateurs d'art à Paris au XVIII siècle, Seyssel, Champ Vallon, 2008 et «Taste communities. The Rise of the Amateur in Eighteenth-Century Paris », EighteenthCentury Studies, 45- 4, été 2012, p. 519-547. 
penser la société. Cette catégorie désigne des individus venus d'horizons sociaux différents (financiers, noblesse de robe et d'épée, marchands), soudés par une passion commune. L'amour de l'art et des sciences rend possible une forme de fluidité sociale, même éphémère et fragile, toujours en performance, à distance des hiérarchies de statuts qui caractérisent la société d'Ancien Régime. Cette lecture pragmatique invite à comprendre autrement le lien entre les productions culturelles et scientifiques et la société, à partir d'une échelle plus fine du social, en partant des objets et des œuvres, et des associations qu'ils peuvent produire, et non pas des hiérarchies sociales surplombantes et prédéfinies et permet de comprendre que, au-delà des milieux restreints de l'Académie, ces transformations participent d'une place nouvelle de l'art dans la société. Un exemple intéressant est la carte adresse du marchand mercier parisien Edme-François Gersaint (dont la boutique a été rendue célèbre par le tableau de Watteau). Dessinée par François Boucher, avant d'être gravée et signée par le comte de Caylus, membre de plusieurs académies, graveur amateur et noble d'ancienne lignée, cette carte avait aussi une fonction commerciale et circulait dans l'espace urbain de la capitale. Une telle image, qui publie l'amitié et les liens entre deux hommes qui appartiennent a priori à deux univers sociaux très différents, n'est possible qu'en raison de leur passion partagée pour l'art et les images d'art. Ici, l'art a la capacité de transformer le social.

Plus concrètement, le nombre croissant des artistes amateurs en Europe au XVIII ${ }^{\mathrm{e}}$ siècle témoigne de nouvelles pratiques de l'image et d'un nouveau rapport au paysage. Le dessin devient un moyen d'exploration du monde et de la subjectivité. Ainsi, les croquis et dessins réalisés durant le temps du voyage, sur le vif, au crayon ou à l'aquarelle, se multiplient et témoignent du développement de cette appropriation visuelle de la nature, plus sensible et plus personnelle. En 1804, est fondée à Londres la Society of Painters in Watercolour qui reconnait à l'aquarelle la dignité d'un genre artistique autonome. Avec cette nouvelle culture matérielle de l'artiste amateur (livres et manuels à dessiner, crayons de couleur inventés par Nadaux, papier à dessiner, petit nécessaire de peintre), c'est la place de l'individu dans son environnement naturel qui est sensiblement modifiée.

\section{Richard Taws}

En s'engageant dans la culture visuelle, les révolutionnaires de toutes les convictions se sont situés par rapport à la politique révolutionnaire. Paradoxalement, les produits de l'État révolutionnaire en apparence standardisés, mais pourtant très visuels, nous donnent un point de vue intéressant sur cette question. Les fêtes révolutionnaires, par exemple, 
où l'aspect visuel était à la fois thématisé et reconstitué, ou l'appareil bureaucratique national en pleine expansion avec ses certificats, passeports, formulaires et vignettes, ouvrent la voie à une expérience visuelle quotidienne fascinante. Si la production d'images en grande quantité par le corps législatif révolutionnaire nous donne une bonne idée de ce que les gouvernements successifs souhaitaient faire savoir ou changer au sein de la population française, d'une certaine manière, cela nous permet également de comprendre comment les citoyens répondaient à l'État révolutionnaire et d'avoir accès à ce qu'ils reconnaissaient ou subvertissaient dans les images réalisées en leur nom. Forger, plier, attacher et adapter, collecter et porter, remodeler et refaire étaient autant de stratégies grâce auxquelles les citoyens pouvaient transformer la fonction et l'apparence des images et des objets qu'ils rencontraient.

C'est ainsi que les confrontations à des images visuelles, qui se passaient souvent à un niveau plutôt personnel, étaient un moyen pour les individus de comprendre leur rôle dans la société pendant la Révolution française. Il nous est bien entendu souvent impossible de reconstituer ces utilisations (et abus) d'images dans leur intégralité. Nous pouvons supposer par exemple que les surfaces intensément travaillées de la monnaie papier révolutionnaire, avec ses filigranes, ses cachets, dates et signatures, ont pu pousser ceux qui les détenaient à y porter une attention particulière. Tentant de distinguer les vrais assignats des contrefaçons, ceux qui les utilisaient devaient, nous pouvons le supposer, les porter à la lumière pour examiner leur conception, entrant ainsi en contact sur le plan somatique avec des éléments du symbolisme révolutionnaire. D'autres images nous montrent que cela se passait ainsi, à coup sûr, mais nous ne pouvons accéder directement à cette expérience. Néanmoins, les critères révolutionnaires de la vertu politique, l'exigence de « transparence » dans tous les domaines de la vie publique et privée, se sont possiblement reflétés de manière intéressante dans ce type de pratiques et ces objets quotidiens faisaient partie des images révolutionnaires qui ont le plus circulé, touchant un public bien plus grand que l'art académique de niveau supérieur. Pour Jonathan Crary, le début du dix-neuvième siècle a vu l'émergence d'une importante individualisation de l'expérience visuelle à l'aide des nouvelles techniques visuelles (les zootropes, les lanternes magiques, etc.), qui peut être mise en parallèle avec l'atomisation des sujets individuels dans la modernité. Je ne me risquerais pas à émettre ce type d'affirmations panoramiques pour la Révolution française, mais il est certain que la culture visuelle a été l'un des premiers domaines grâce auxquels nous avons pu observer la relation entre les individus et la société et qu'elle a été essentielle pour comprendre 
la manière dont les révolutionnaires (et ceux qui se sont opposés à la Révolution) ont compris ce que cela signifiait d'être « révolutionnaire».

Peut-on parler d'une révolution de l'œil qui précéderait ou accompagnerait les révolutions entre les années 1770 et 1830 ?

\section{Richard Taws}

La Révolution française a sans aucun doute changé la manière dont la population envisageait le monde et par la suite, rien ne pouvait plus vraiment être perçu avec le même regard. Comme je l'ai déjà laissé entendre, je dirais que les images ont joué un rôle essentiel dans ce processus, même si ce ne fut pas toujours de manière directe, et pas facilement liée à des transformations plus générales dans la compréhension de la vision. Toutefois, les attentes placées dans les images au cours des années 1790 ont pris une forme très différente aux alentours de 1830 , car, à cette époque, les comportements acquis concernant la pratique visuelle dans un contexte révolutionnaire ont radicalement changé ce que les individus attendaient des images, ainsi que la forme qu'elles ont prise. En réalité, pour expliquer la relation dialectique entre les pratiques visuelles et la politique révolutionnaire, il apparaît crucial de reconnaître également les identités souvent distinctes des pratiques artistiques et de la politique révolutionnaire dans leurs multiples formes. Chacune disposait de ses propres institutions qui ont évolué en suivant différentes trajectoires au cours de cette période, ces trajectoires se sont souvent croisées, mais n'étaient pas toujours associées l'une à l'autre. En outre, il est important de reconnaître la diversité des éléments pouvant être considérés comme faisant partie de la pratique visuelle « révolutionnaire ». «L'art en période de révolution » n'est pas toujours similaire à l' « art révolutionnaire ». Tout le monde ne regardait pas de la même façon ou aux mêmes fins, même si le regard se portait sur la même chose. Plutôt que de parler d'une « révolution de l'œil » unique qui aurait précédé ou accompagné l'âge des révolutions (il est vrai que le moment, que ce soit avant ou pendant, est crucial), il serait peut-être plus judicieux et historiquement justifié d'envisager un certain nombre de « révolutions » dans les domaines de la technologie, de l'apparence, de la production et du public, chacun de ces éléments présentant également une possibilité de retranchement, de « contre-révolution » et d'échec, ainsi que de progrès et de transformation.

\section{Charlotte Guichard}

Incontestablement, on note un changement du rapport aux images. L'historien de l'art Thomas Crow a soutenu l'idée d'un éveil concomitant 
de la population parisienne à l'esthétique et à la politisation à partir des années 1780. La fréquentation des Salons du Louvre, gratuits et ouverts à tous, culmine ainsi en 1781 avec environ 35000 visiteurs, soit mille personnes par jour, attestant ainsi l'importance culturelle et politique de cet événement artistique dans la capitale. Exposée à chaque salon, la peinture de Jacques-Louis David, qui propose un nouveau modèle civique, moral et politique, suscite la publication de nombreuses brochures et favorise la naissance d'un espace public et politique de la peinture. En France, les travaux des historiens et des historiens de l'art se sont d'abord portés sur l'iconoclasme révolutionnaire et les réactions opposées aux artefacts du passé. Le «partage du patrimoine », selon les mots de Dominique Poulot, témoigne d'un regard nouveau porté sur les artefacts et les images : en 1792, la statue équestre de Louis XIV située place de la Concorde est détruite par les révolutionnaires, alors même que les amateurs regrettent de leur côté la destruction d'une statue possédant une valeur artistique et patrimoniale. Voilà un objet contesté, dont les évaluations sont controversées. Ce qu'il faut bien comprendre, c'est que cette transformation du rapport aux images ne se limite nullement au domaine artistique et à la culture savante des connaisseurs, ou aux usages immédiatement politiques de la peinture d'histoire qui propose des modèles d'identification. En réalité, comme l'a montré récemment Richard Taws, la culture visuelle participe activement à la fabrique de l'idéologie révolutionnaire, à travers de nouveaux supports de communication et de circulation de l'information que sont les objets visuels comme les calendriers, les almanachs, les assignats, etc. De ce point de vue, le cas de la France révolutionnaire est assez proche des révolutions atlantiques.

Les cultures visuelles des révolutions atlantiques témoignent en effet d'un élargissement de la gamme des supports visuels de l'idéologie révolutionnaire. La grande peinture d'histoire, incarnée par Benjamin West, John Singleton Copley ou John Trumbull, véhicule des images patriotiques susceptibles de fonder de nouvelles communautés nationales, où les questions de la race et de l'empire, de la nation et de la métropole se croisent dans l'image. Mais d'autres supports plus inattendus, comme le homespun étudié par Leora Auslander, montrent aussi combien la fabrique de l'image s'inscrit dans des pratiques sociales, des communautés domestiques et locales, et des situations de genre. La guerre d'Indépendance américaine et les premiers temps de la jeune République américaine offrent de ce point de vue un aperçu très riche et surprenant sur les rapports entre cultures visuelles et grande peinture d'histoire, entre peinture et nouvelles technologies comme la télégraphie et le daguerréotype (cf. la 
figure de Samuel Morse). Il s'agit d'un champ en plein essor dans le monde anglophone, moins développé en France, qui vise à montrer les liens complexes qui existent entre trois niveaux de transformations : la multiplication des images et de leurs supports, qui relève d'une mutation technique et matérielle, les nouvelles cultures visuelles, qui impliquent de nouveaux regards portés sur l'image, et les transformations politiques associées à ce que l'on peut appeler l'ère des révolutions, de la fin du $\mathrm{XVIII}^{\mathrm{e}}$ au milieu du XIX ${ }^{\mathrm{e}}$ siècle.

\section{Pascal Griener}

Comme je l'ai signalé plus haut, je ne crois nullement que les révolutions de l'œil et les révolutions politiques appartiennent à la même temporalité, ni qu'elles touchent les mêmes catégories sociales. Le croire, c'est se contenter d'un modèle simpliste, régi par une temporalité lisse, toujours progressive. Aujourd'hui, il ne me semble plus possible de céder à cette illusion.

Guillaume MAZEAU Université de Paris I-Panthéon-Sorbonne/IHRF mazeau.guillaume@free.fr

Pascal DuPUY Université de Rouen/GRHIS pascaldupuy@hotmail.com

Charlotte GUICHARD Chargée de recherche au CNRS UMR 8529/Université Lille-3 charlotte.guichard@wanadoo.fr

Richard TAWS

History of Art Department, University College London r.taws@ucl.ac.uk

Pascal GRIENER D. Phil Oxford, Institut d'Histoire de l'Art \& de Muséologie, Université de Neuchâtel pascal.griener@unine.ch 